\title{
Hsa_circ_0001649: A circular RNA and potential novel biomarker for hepatocellular carcinoma
}

\author{
Meilin Qin ${ }^{\mathrm{a}, \mathrm{b}, 1}$, Gang Liu ${ }^{\mathrm{c}, 1}$, Xisong Huo ${ }^{\mathrm{b}}$, Xuemei Tao ${ }^{\mathrm{b}}$, Xiaomeng Sun ${ }^{\mathrm{a}}$, Zhouhong Ge ${ }^{\mathrm{b}}$, Juan Yang ${ }^{\mathrm{d}}$, \\ Jia Fan ${ }^{\mathrm{e}}$, Lei Liu ${ }^{\mathrm{a}, *}$ and Wenxin Qin ${ }^{\mathrm{b}, *}$ \\ ${ }^{a}$ Shanghai Medical College of Fudan University, Shanghai, China \\ ${ }^{\mathrm{b}}$ State Key Laboratory of Oncogenes and Related Genes, Shanghai Cancer Institute, Renji Hospital, Shanghai Jiao \\ Tong University School of Medicine, Shanghai, China \\ ${ }^{\mathrm{c}}$ Key Laboratory of Systems Biology, Institute of Biochemistry and Cell Biology, Shanghai Institutes for Biological \\ Sciences, China Academy of Scieneces, Shanghai, China \\ ${ }^{\mathrm{d}}$ Medical College of Nantong University, Nantong, Jiangsu, China \\ ${ }^{\mathrm{e}}$ Liver Cancer Institute, Zhongshan Hospital and Shanghai Medical College, Fudan University, Key Laboratory for \\ Carcinogenesis and Cancer Invasion, the Chinese Ministry of Education, Shanghai, China
}

\begin{abstract}
.
BACKGROUND: It has been shown that circular RNA (circRNA) is associated with human cancers, however, few studies have been reported in hepatocellular carcinoma (HCC).

OBJECTIVE: To estimate clinical values of a circular RNA, Hsa_circ_0001649, in HCC.

METHODS: Expression level of hsa_circ_0001649 was detected in HCC and paired adjacent liver tissues by real-time quantitative reverse transcription-polymerase chain reactions (qRT-PCRs). Differences in expression level of hsa_circ_0001649 were analyzed using the paired t-test. Tests were performed between clinical information and hsa_circ_0001649 expression level by analysis of variance (ANOVA) or welch t-test and a receiver operating characteristics (ROC) curve was established to estimate the value of hsa_circ_0001649 expression as a biomarker in HCC.

RESULTS: hsa_circ_0001649 expression was significantly downregulated in HCC tissues $(p=0.0014)$ based on an analysis of 89 paired samples of HCC and adjacent liver tissues and the area under the ROC curve (AUC) was 0.63. Furthermore, hsa_circ_0001649 expression was correlated with tumor size $(p=0.045)$ and the occurrence of tumor embolus $(p=0.017)$ in HCC.

CONCLUSIONS: We first found hsa_circ_0001649 was significantly downregulated in HCC. Our findings indicate hsa_circ_0001649 might serve as a novel potential biomarker for HCC and may function in tumorigenesis and metastasis of HCC.
\end{abstract}

Keywords: Circular RNA, hsa_circ_0001649, hsa_circ_001599, hepatocellular carcinoma, biomarker

\footnotetext{
${ }^{1}$ These authors contributed equally to this work.

* Corresponding authors: Wenxin Qin, State Key Laboratory of Oncogenes and Related Genes, Shanghai Cancer Institute, Renji Hospital, Shanghai Jiao Tong University School of Medicine, No. 25/Ln 2200 Xie-Tu Road, Shanghai 200032, China. E-mail: wxqin@sjtu.edu.cn; Lei Liu, Institutes for Biomedical Sciences, Fudan University, No. 131 Dongan Road, Shanghai, 200032, China. E-mail: liulei@fudan.edu.cn.
}

\section{Abbreviations}

CircRNA， Circular RNA;

HCC, Hepatocellular carcinoma;

ROC, Receiver operating characteristics;

ANOVA, Analysis of variance;

AUC, Area under the ROC curve;

HDV, Hepatitis delta virus; 
SHPRH, SNF2 histone linker PHD RING helicase;

MMP, Matrix metalloproteinase;

EMT, Epithelial mesenchymal transition;

RBP, RNA-binding protein;

U2AF65, U2 auxiliary factor $65 \mathrm{kDa}$ subunit;

EIF4A3, Eukaryotic initiation factor 4A-III;

UPF1, Regulator of nonsense transcripts 1 .

\section{Introduction}

Circular RNAs (CircRNAs) are class of RNAs formed by back-splicing events as loops, and are found in all types of organisms. They have been identified in viroidal plant pathogens and the hepatitis delta virus (HDV) [12], and described as spliced tRNAs, rRNA introns, and as rRNA processing intermediates in archaea [34]. CircRNAs in eukaryotes were initially detected in 1979, and then in mouse in 1993 [56].

In the early decades, circRNAs were thought to be expressed at so low levels that they have been generally regarded as byproducts of splicing errors [7], and have not been regarded as biologically active regulators. However, the advent of next generation sequencing has illuminated circRNAs as an entire class of abundant, non-coding RNAs ubiquitous among eukaryotes [8,9] and several circRNAs has been identified and reported to have physiological functions recently. CircRNAs can act as miRNA sponges [8 $[10]$ and can function in gene regulation as transcription regulators interacting with the Pol II transcription complex [11] or by competing with linear splicing [12]. Other investigators have reported that circRNAs are involved in the growth of colorectal and ovarian cancers [13], and be associated with DNA damage in breast cancer cells [14].

CircRNAs have recently been described as biomarkers of aging in Drosophila [15] and disease biomarkers in human saliva [16]. Additionally, circRNA has been suggested as a potential novel biomarker for use in diagnosing cancers [17/18]. Hepatocellular carcinoma (HCC) is the third most common cause of cancerrelated death world-wide [19], and the majority of HCC patients have a poor prognosis due to the high frequency of disease metastasis and recurrence [20[21]. Therefore, it is critical to find novel biomarkers for improving the prognosis and therapy of HCC. In this study, we focused on a circRNA, hsa_circ_0001649 (ID: hsa_circ_0001649), from database CircBase, because hsa_circ_0001649has been predicted to be asso- ciated with HCC in circ2Traits (ID:hsa_circ_001599 in database circ2Traits) [22]. We first verified hsa_circ_ 0001649 expression was significantly downregulated in HCC tissues. Our results indicate that hsa_circ_000 1649 may serve as a novel potential biomarker for HCC and may play roles in tumorigenesis and metastasis of HCC.

\section{Materials and methods}

\subsection{Samples}

The 89 samples of HCC and paired adjacent liver tissues were obtained from Zhongshan Hospital (Shanghai, China). All tissue specimens were stored at $-80^{\circ} \mathrm{C}$ until use. Clinical information was obtained under an Institutional Review Board approved study protocol and written informed consent was obtained from each subject.

\subsection{Cell culture}

Immortalized hepatoma cell lines, HCC-LM3 and MHCC-97L, were from Zhongshan Hospital of Shanghai Medical College, Fudan University [2324]. All cells were cultured in Dulbecco's modified Eagle's medium (DMEM) (Gibco, Gaithersburg, MD, USA) containing $10 \%$ fetal bovine serum (FBS) and a humidified atmosphere consisting of $5 \% \mathrm{CO}_{2}$ and $95 \%$ air at $37^{\circ} \mathrm{C}$.

\subsection{Total RNA extraction}

Total RNA was extracted from each sample of hepatocellular carcinoma and paired paracancerous liver tissue using TRIzol Reagent (Invitrogen; Carlsbad, CA, USA) following the manufacturer's instructions.

\subsection{Reverse transcription}

The cDNA was synthesized by reverse transcription (RT) using a Primescipt RT reagent kit with gRNA Eraser according to manufacture-provided protocols (TaKaRa) (with random primers).

\subsection{Real-time polymerase chain reaction assay}

Real-time quantitative reverse transcription-polymerase chain reactions (qRT-PCR) were performed us- 
Table 1

qRT-PCR primer sequences

\begin{tabular}{lll}
\hline Primer name & Primer FW $\left(5^{\prime}-3^{\prime}\right)$ & Primer RV $\left(5^{\prime}-3^{\prime}\right)$ \\
\hline Hsa_circ_0001649 & AATGCTGAAAACTGCTGAGAGAA & TTGAGAAAACGAGTGCTTTGG \\
$\beta$-actin & AGTTGCGTTACACCCTTTCTTG & GCTGTCACCTTCACCGTTCC \\
SHPRH & ATGAGCAGCCGACGGAAACG & ATCTGAACCTGGGCAGGGCT \\
MMP9 & GCCTTCGCACTGTGGAGC & GGATACCCGTCTCCGTGCTC \\
MMP10 & CACTGGAACCCTGAACCTGAAT & AATAAAAACGGTGTCCCTGCTG \\
MMP2 & ATGCCGTCGTGGACCTGC & TGCTTCCAAACTTCACGCTCTT \\
MMP13 & TCTTTCTTCGGCTTAGAGGTGACT & AAACATTGTATTCACCCACATCAGG \\
MMP7 & CGATGAGGATGAACGCTGGA & AGGAATGTCCCATACCCAAAGAA \\
\hline
\end{tabular}

ing SYBR ${ }^{\circledR}$ Premix Ex Taq ${ }^{\mathrm{TM}}$ II (Tli RNaseH Plus) (TaKaRa), following manufacturer's instructions. Divergent primers for hsa_circ_0001649 and primers for $\beta$-actin [25], SHPRH, MMP9, MMP10, MMP2, MMP13, as well as MMP7 were synthesized by Sangon Biotech (Shanghai, China). All qRT-PCR primer sequences see Table 1.

\subsection{Sanger sequencing}

The target fragment was inserted into a $\mathrm{T}$ vector for Sanger sequencing to determine its full-length. The following divergent primers were designed for use in experiments to confirm the back-splice junction of hsa_circ_0001649: 5'-CAATGCTGAAAACTGCTG AGAGAAG-3' (sense) and 5'-CCTGCATTCTTTCTT CTATTGTTGCTTTAA-3' (antisense). The primers were synthesized by Sangon Biotech (Shanghai, China), and Sanger sequencing was performed by Biosune (Shanghai, China).

\section{7. siRNA interference}

siRNA of hsa_circ_0001649 was designed and synthesized by GenePharma (Shanghai, China), targeting to the junction region of hsa_circ_0001649 sequence. The siRNA sequences were as follows: 5'UGGCUGCCCUUCUCUCAGCTT-3' (sense) and 5'GCUGAGAGAAGGGCAGCCATT-3' (antisense).

\subsection{Statistical analysis}

All data analyses were performed with R (v 2.15.0, http://www.r-project.org/). Differences in values for hsa_circ_0001649 were based on comparisons of its expression in $89 \mathrm{HCC}$ tissues and their paired paracancerous liver tissues as determined using the paired t-test. Analysis of variance (ANOVA) was used to determine correlations between expression levels of hsa_circ_0001649 and clinical indexes. A receiver operating characteristics (ROC) curve for hsa_circ_0001
649 in detecting HCC was drawn using "ROCR" (http://cran.r-project.org/web/packages/ROCR/). Pvalues $<0.05$ were considered statistically significant.

\section{Results}

\subsection{Detection of naturally existing hsa_circ_0001649 in HCC tissues}

Hsa_circ_0001649 is produced at the SHPRH gene locus containing exon 26-29 (Fig. 1A). Results of RT-PCR performed with divergent primers indicated that hsa_circ_0001649 was expressed in samples of HCC tissues (Fig. 1B), and sequencing of the RTPCR product of hsa_circ_0001649 showed a fulllength sequence of hsa_circ_0001649 that was completely consistent with that in CircBase. Results of the Sanger sequencing experiment confirmed the backsplice junction of hsa_circ_0001649 (Fig. 1C), proving that hsa_circ_0001649 naturally existed as a loop in HCC tissues.

\subsection{Downregulation of hsa_circ_0001649 in HCC tissues}

We detected the expression levels of hsa_circ_0001 649 in 89 samples of HCC tissues and their paired adjacent liver tissues by qRT-PCR, when using $\beta$ actin as the internal standard. Our results showed hsa_circ_0001649 expression in HCC tissues was significantly lower compared to its expression in the paired adjacent liver tissues ( $n=89 ; p=0.0014$ ) (Fig. 2A). The relative expression level of hsa_circ_ 0001649 in each sample of HCC and paired adjacent liver tissue is shown in Fig. 2B. The specificity of the qRT-PCR product of hsa_circ_0001649 was analyzed by electrophoresis, and the amplified product yielded a single peak in a melting curve analysis (Supplementary Fig. 1). 


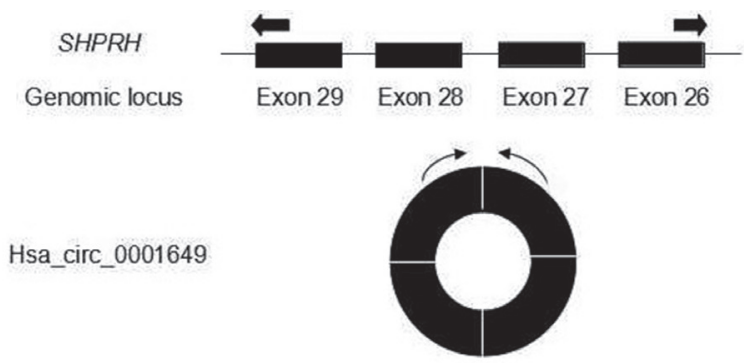

A
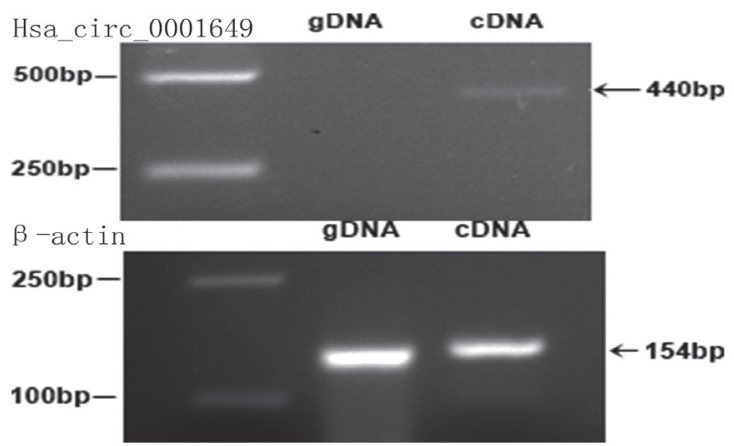

B

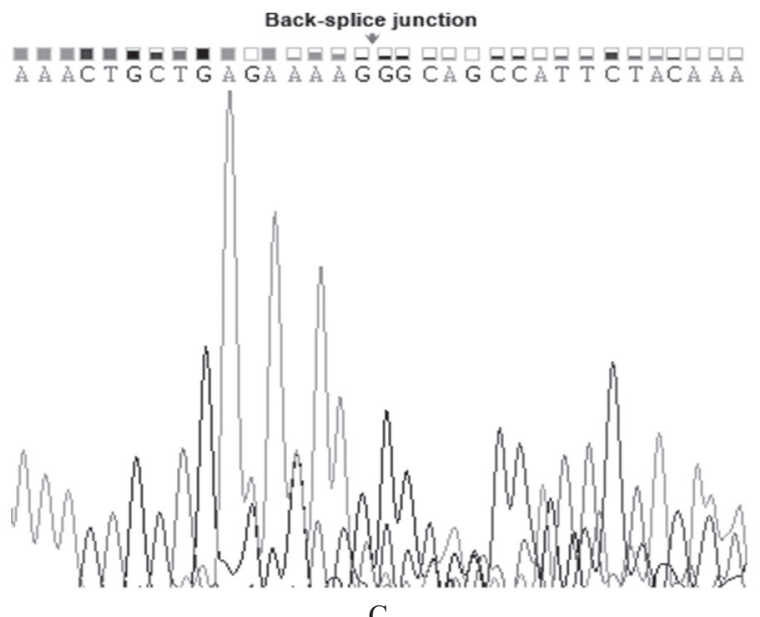

Fig. 1. Identification of hsa_circ_0001649 in samples of HCC tissues. (A) Hsa_circ_0001649 is produced at the SHPRH gene locus containing exon 26-29. (B) RT-PCR products with divergent primers of hsa_circ_0001649 in HCC-LM3 cells verified that hsa_circ_0001649 naturally existed in HCC tissues. (C) Sanger sequencing of hsa_circ_0001649 showed the back-splice junction.

\subsection{Potential values of hsa_circ_0001649 as a biomarker for HCC}

Our results showed that hsa_circ_0001649 expression was significantly downregulated in samples of HCC tissue, and we therefore examined our data for potential correlations between hsa_circ_0001649 ex-
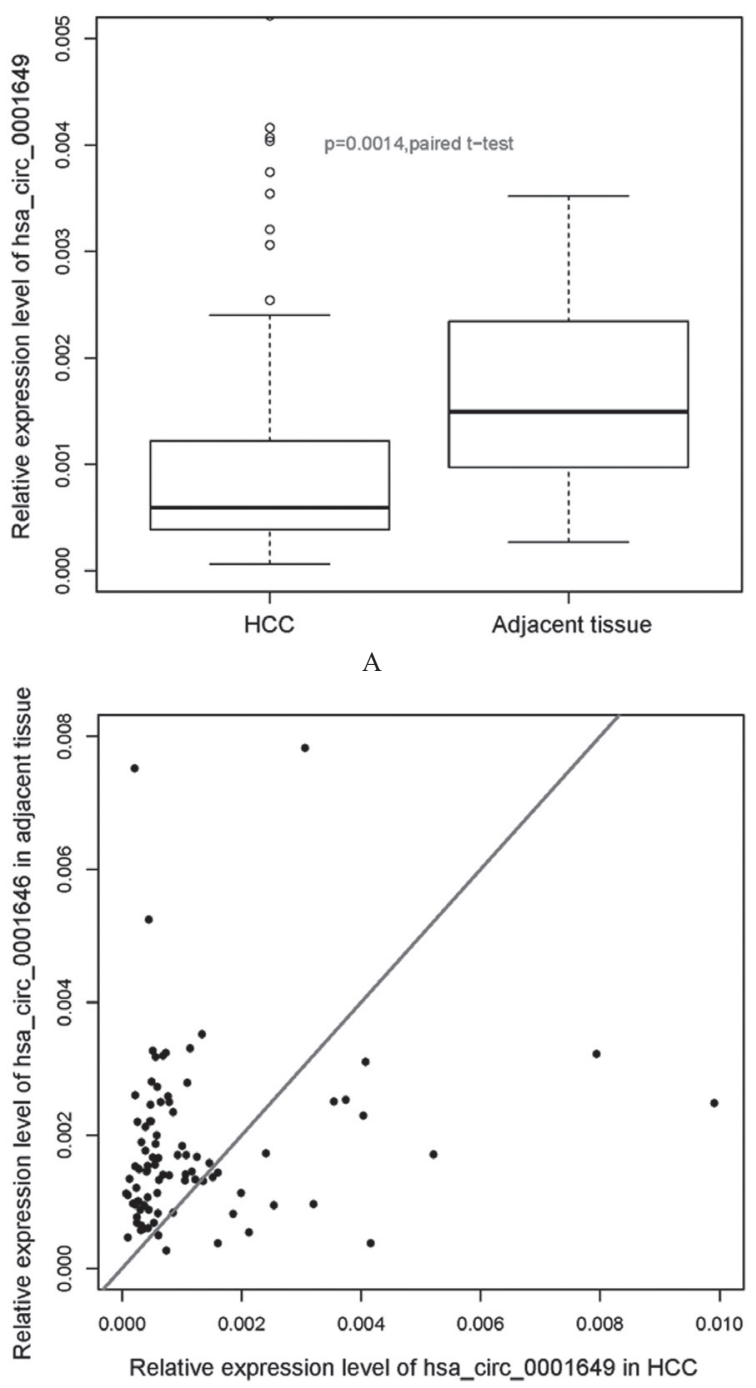

B

Fig. 2. Downregulation of hsa_circ_0001649 in HCC tissues. (A) Expression differences of hsa_circ_0001649 between HCC and matched adjacent liver tissues $(n=89, p=0.0014)$. (B) The relative expression level of hsa_circ_0001649 in each HCC and paired adjacent liver tissue. Dots above the line are samples with downregulated expression of hsa_circ_0001649.

pression and clinical parameters. We found that tumor size was related to the level of hsa_circ_0001649 expression ( $p=0.045$ ) (Table 2). Additionally, tumor embolus was significantly related to the level of hsa_circ_0001649 expression by welch t-test ( $p=$ 0.017) (Fig. 3). The occurrence of tumor embolus reflects invasion status of cancer cells and indicates the metastatic possibility, so we made further research to investigate potential correlations between hsa_circ_0001649 expression and metastasis. And after knockdown of hsa_circ_0001649 with siRNA in 
Table 2

Correlation between hsa_circ_0001649 expression and clinical parameters in HCC

\begin{tabular}{|c|c|c|c|}
\hline Characteristics & Patient number & Mean $\pm \mathrm{SD}$ & $P$ value \\
\hline Gender & & & 0.88202 \\
\hline Female & 15 & $0.00113 \pm 0.00149$ & \\
\hline Male & 74 & $0.00120 \pm 0.00162$ & \\
\hline Age & & & 0.96582 \\
\hline$\leqslant 60$ & 58 & $0.00118 \pm 0.00178$ & \\
\hline$>60$ & 31 & $0.00119 \pm 0.00119$ & \\
\hline Cirhosis_history & & & 0.78937 \\
\hline Negative & 15 & $0.00109 \pm 0.0014$ & \\
\hline Positive & 75 & $0.00121 \pm 0.00164$ & \\
\hline HCC_history & & & 0.51943 \\
\hline Negative & 80 & $0.00122 \pm 0.00167$ & \\
\hline Positive & 9 & $0.00086 \pm 0.00045$ & \\
\hline HBsAg & & & 0.81243 \\
\hline Negative & 23 & $0.00112 \pm 0.00108$ & \\
\hline Positive & 66 & $0.00121 \pm 0.00174$ & \\
\hline $\mathrm{HBcAb}$ & & & 0.4407 \\
\hline Negative & 11 & $0.00153 \pm 0.00138$ & \\
\hline Positive & 78 & $0.00114 \pm 0.00162$ & \\
\hline Cirhosis_nodes & & & 0.98257 \\
\hline '1 & 21 & $0.00118 \pm 0.00208$ & \\
\hline$>1$ & 68 & $0.00119 \pm 0.00143$ & \\
\hline Membrane & & & 0.38813 \\
\hline Negative & 41 & $0.00103 \pm 0.00123$ & \\
\hline Positive & 48 & $0.00132 \pm 0.00185$ & \\
\hline Events & & & 0.1144 \\
\hline Negative & 40 & $0.00148 \pm 0.00208$ & \\
\hline Positive & 49 & $0.00094 \pm 0.00099$ & \\
\hline Recurrence & & & 0.62813 \\
\hline Negative & 44 & $0.00127 \pm 0.00175$ & \\
\hline Positive & 45 & $0.0011 \pm 0.00143$ & \\
\hline Embolus & & & 0.05239 \\
\hline Negative & 58 & $0.00142 \pm 0.00185$ & \\
\hline Positive & 31 & $0.00074 \pm 8 \mathrm{e}-04$ & \\
\hline Tumor size & & & $0.04512^{*}$ \\
\hline$\leqslant 5 \mathrm{~cm}$ & 50 & $0.00148 \pm 0.00198$ & \\
\hline$>5 \mathrm{~cm}$ & 39 & $8 \mathrm{e}-04 \pm 0.00074$ & \\
\hline Tumor number & & & 0.45038 \\
\hline 1 & 73 & $0.00112 \pm 0.00135$ & \\
\hline$>1$ & 16 & $0.00146 \pm 0.00244$ & \\
\hline AFP1 & & & 0.55783 \\
\hline Negative & 32 & $0.00132 \pm 0.00164$ & \\
\hline Positive & 57 & $0.00111 \pm 0.00158$ & \\
\hline AFP2 & & & 0.29426 \\
\hline Negative & 54 & $0.00133 \pm 0.00184$ & \\
\hline Positive & 35 & $0.00096 \pm 0.0011$ & \\
\hline GGT & & & 0.27857 \\
\hline Negative & 35 & $0.00141 \pm 0.00218$ & \\
\hline Positive & 54 & $0.00104 \pm 0.00105$ & \\
\hline ALT & & & 0.80302 \\
\hline$\leqslant 54$ & 76 & $0.00117 \pm 0.00167$ & \\
\hline$>54$ & 13 & $0.00129 \pm 0.0011$ & \\
\hline Differentiation & & & 0.30002 \\
\hline $\mathrm{I}+\mathrm{II}$ & 71 & $0.00127 \pm 0.00172$ & \\
\hline $\mathrm{III}+\mathrm{IV}$ & 19 & $0.00083 \pm 0.00095$ & \\
\hline TNM & & & 0.57101 \\
\hline Early & 51 & $0.00127 \pm 0.00149$ & \\
\hline Late & 38 & $0.00107 \pm 0.00173$ & \\
\hline BCLC & & & 0.13539 \\
\hline $0+\mathrm{A}$ & 19 & $0.00167 \pm 0.00208$ & \\
\hline $\mathrm{B}+\mathrm{C}+\mathrm{D}$ & 70 & $0.00105 \pm 0.00142$ & \\
\hline
\end{tabular}




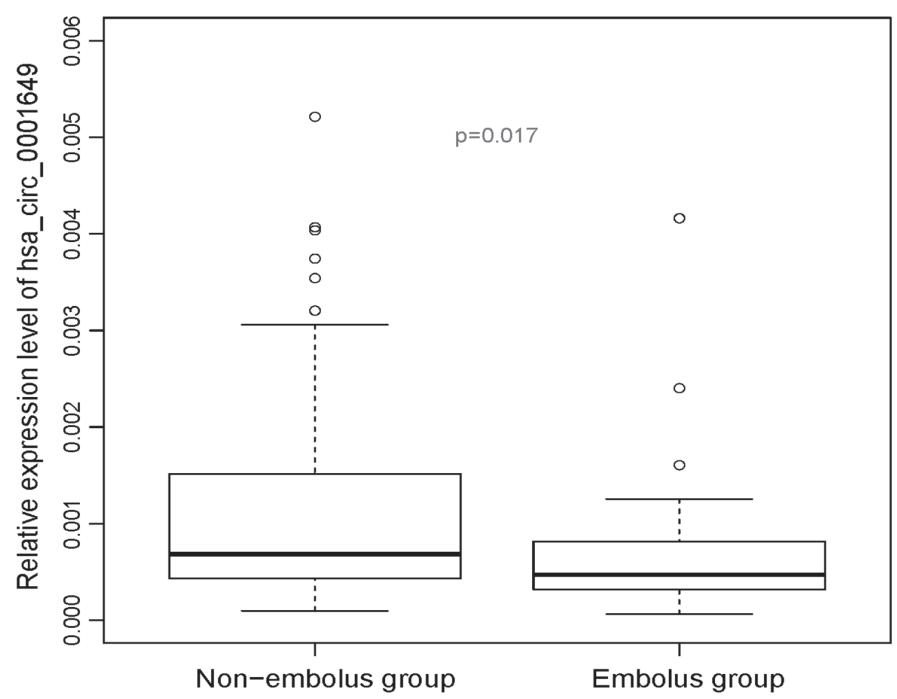

Fig. 3. Hsa_circ_0001649 expression was correlated with tumor embolus in HCC ( $p=0.017)$. The expression level of has_circ0001649 is significantly lower in the samples with embolus $(n=31)$ than those without embolus $(n=58)$ according to welch t-test. The occurrence of tumor embolus reflects invasion status of cancer cells.
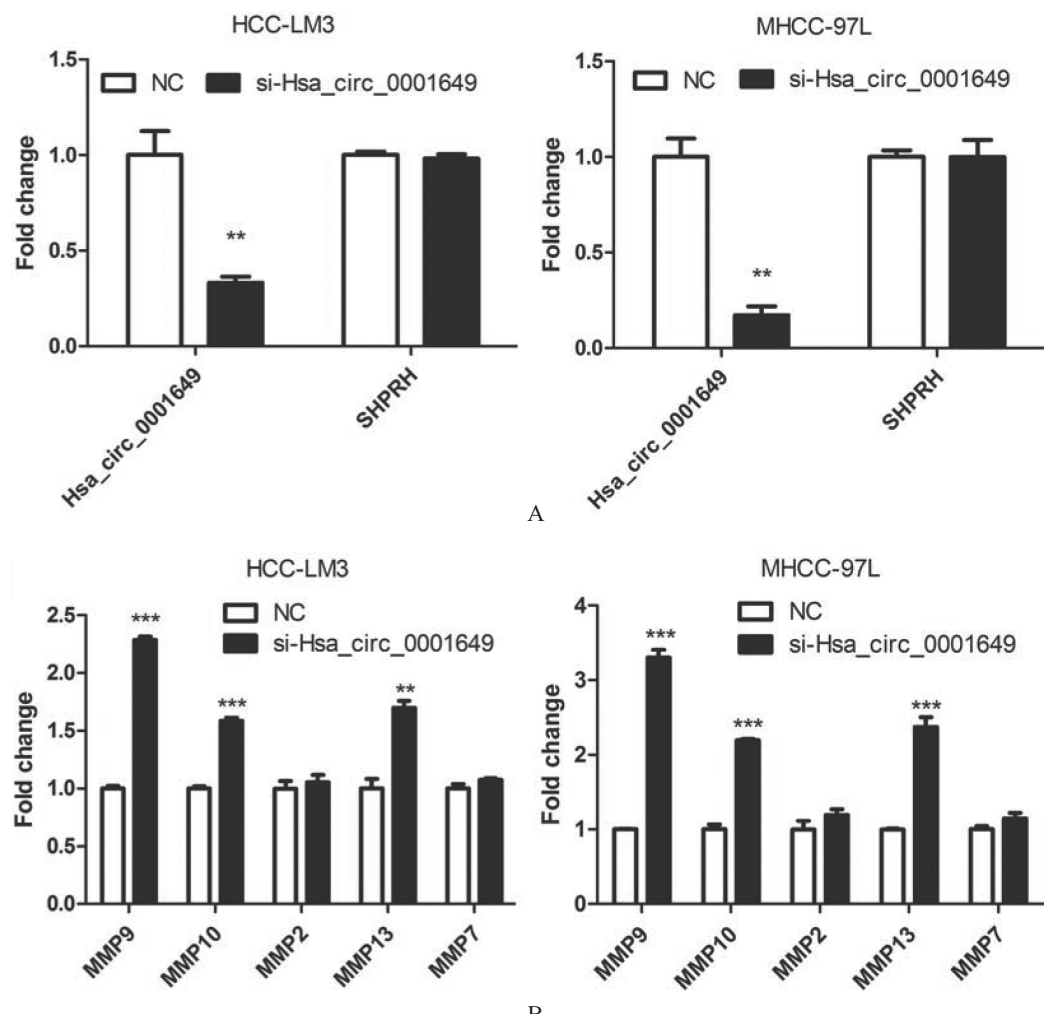

Fig. 4. Hsa_circ_0001649 expression is associated with metastasis in HCC. (A) Effective knockdown of hsa_circ_0001649 with siRNA. It avoided interfering with the expression of linear mRNA SHPRH, both in HCC-LM3 cells and MHCC-97L cells. The siRNA targeted the junction sequences of hsa_circ_0001649. (B) Increase in mRNA levels of MMP9, MMP10, and MMP13 after knockdown of hsa_circ_0001649 with siRNA in HCC-LM3 cells and MHCC-97L cells (P-values $<0.01)$. 


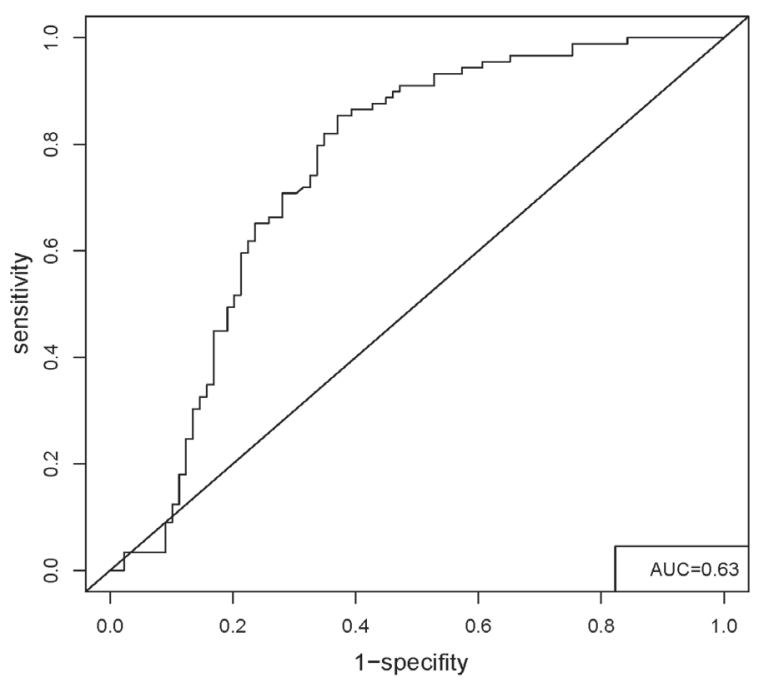

A

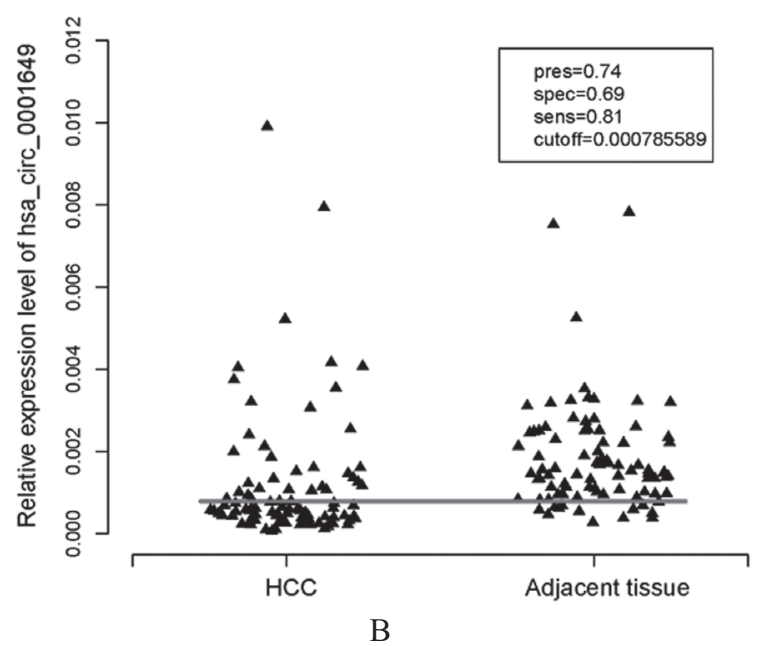

Fig. 5. Hsa_circ_0001649 might serve as a biomarker for HCC. (A) A larger AUC by ROC analysis for hsa_circ_0001649 in HCC indicates greater potential as a biomarker ( $\mathrm{AUC}=0.63$ ). (B) The cutoff value, sensitivity, and specificity were $0.0007855,0.81$, and 0.69 , respectively. Dots above the line predict areas of adjacent non-cancerous tissue and dots below the line predict cancerous tissue.

immortalized hepatoma cell lines, HCC-LM3 and MHCC-97L (Fig. 4A), we tested the expression levels of several matrix metallopeptidases (MMPs) 9, 10, 2,13 , and 7 , which had been reported to have key roles in promoting the metastasis of HCC [26-29]. We found significant increase in mRNA levels of MMP9, MMP10, and MMP13 (Fig. 4B) which indicated that low level of hsa_circ_0001649 expression was positively correlated with the metastasis of HCC.

We next investigated whether hsa_circ_0001649 expression could serve as a biomarker for distinguishing cancerous tissue from adjacent non-cancerous liver tissue. When expression levels of hsa_circ_0001649 were examined for this purpose, the area under the ROC curve (AUC) was 0.63. (Fig. 5A), and the cutoff value, sensitivity, and specificity were 0.0007855 , 0.81, and 0.69, respectively (Fig. 5B).

\section{Discussion}

We have shown in this study that the expression level of hsa_circ_0001649 is downregulated in HCC tissues when compared to the matched adjacent liver tissues. The hsa_circ_0001649 expression is related to tumor size $(p=0.045)$. Interestingly, HCC tumors with larger size show lower level of hsa_circ_0001649 expression, suggesting a role for hsa_circ_0001649 in tumor growth. Furthermore, we found expression levels of hsa_circ_0001649 were related to tumor embolus. Moreover, mRNA levels of MMP 9, 10, and 13 were significant increased after knockdown of hsa_circ_0001649 with siRNA in HCC cells. The occurrence of tumor embolus reflects invasion status of cancer cells and MMPs have key roles in promoting the metastasis of HCC. These results indicated that low level of hsa_circ_0001649 expression was positively correlated with the metastasis of $\mathrm{HCC}$ and it may function in metastasis of HCC, however, detailed molecular mechanisms of which hsa_circ_0001649 involved in HCC invasion and metastasis need to be revealed. Our results further suggest downregulation of hsa_circ_0001649 is associated with poor prognosis of HCC and ROC analyses show that hsa_circ_0001649 may be used as a novel biomarker for HCC with high degrees of accuracy, specificity, and sensitivity.

It has been proposed that circRNAs may act as competing endogenous RNAs to bind proteins (protein sponges) [30[31]. Then, we focused on the sequence of hsa_circ_0001649 to find its potential RNA-binding proteins (RBPs) binding sites (starBase v2.0) [32], and we found that hsa_circ_0001649 had 6 potential protein binding sites for U2 auxiliary factor $65 \mathrm{kDa}$ subunit (U2AF65), 5 potential protein binding sites for Eukaryotic initiation factor 4A-III (EIF4A3), and 1 potential protein binding site for Regulator of nonsense transcripts 1 (UPF1). Those analysis results imply that hsa_circ_0001649 may function as a protein sponge or a transcription regulator to participate in development and progression of HCC.

Two circRNAs have been verified as being actual miRNA sponges in mammals so far [10]. One specific circRNA, (ciRS-7, also known as CDR1as), has 
$>70$ binding sites for miR-7, and it has been shown that circRNA impairs the regulatory effect of miR-7 in vivo. The second circRNA is the testis-specific transcript of the male sex-determining gene Sry, which contains 16 binding sites for miR-138. Many of the predicted miRNA binding sites in circRNAs are functional [33]. An additional proposed function of circRNAs is the transport of miRNAs [34]. We found hsa_circ_0001649 had potential for harboring miRNA binding sites (http://www.mirdb.org/miRDB/custom. html) and It possessed potential binding sites for hsamiR-1283, hsa-miR-4310, hsa-miR-182-3p, hsa-miR888-3p, hsa-miR-4502, hsa-miR-6811-5p, hsa-miR6511b-5p, and hsa-miR-1972. Those analysis results suggest that hsa_circ_0001649 may play roles in HCC by interacting with miRNAs.

In summary, we first identified hsa_circ_0001649 expression was significantly downregulated in HCC tissues and it might serve as a novel potential biomarker for HCC. Furthermore, our results indicated that hsa_circ_0001649 might be involved in tumorigenesis and metastasis of HCC.

\section{Acknowledgments}

This work was supported by National Natural Science Fund of China (81302093, 81402278, 81421001), Grants from the State Key Laboratory of Oncogenes and Related Genes (91-1305, 91-1411, and SB14-03), Doctoral Innovation Fund Projects from Shanghai Jiao Tong University School of Medicine (BXJ201319), and Key Discipline and Specialty Foundation of Shanghai Municipal Commission of Health and Family Planning.

\section{References}

[1] Flores R, Grubb D, Elleuch A, Nohales M? Delgado S, Gago S. Rolling-circle replication of viroids, viroid-like satellite RNAs and hepatitis delta virus: Variations on a theme. RNA Biol. 2011; 8: 200-206.

[2] Kos A, Dijkema R, Arnberg AC, van der Meide PH, Schellekens H. The hepatitis delta virus possesses a circular RNA. Nature. 1986; 323: 558-60.

[3] Lykke-Andersen J, Aagaard C, Semionenkov M, Garrett RA. Archaeal introns: Splicing, intercellular mobility and evolution. Trends Biochem Sci. 1997; 22: 326-31.

[4] Salgia SR, Singh SK, Gurha P, Gupta R. Two reactions of Haloferax volcanii RNA splicing enzymes: Joining of exons and circularization of introns. RNA. 2003; 9: 319-30.

[5] Hsu MT, Coca-Prados M. Electron microscopic evidence for the circular form of RNA in the cytoplasm of eukaryotic cells. Nature. 1979; 280: 339-40.
[6] Capel B, Swain A, Nicolis S, Hacker A, Walter M, Koopman P, Goodfellow P, Lovell-Badge R. Circular transcripts of the testis-determining gene Sry in adult mouse testis. Cell. 1993; 73: 1019-30.

[7] Cocquerelle C, Mascrez B, Hétuin D, Bailleul B. Mis-splicing yields circular RNA molecules. FASEB J. 1993; 7: 155-60.

[8] Memczak S, Jens M, Elefsinioti A, Torti F, Krueger J, Rybak A, Maier L, Mackowiak SD, Gregersen LH, Munschauer M, Loewer A, Ziebold U,Landthaler M, Kocks C, le Noble F, Rajewsky N. Circular RNAs are a large class of animal RNAs with regulatory potency. Nature. 2013; 495: 333-8.

[9] Guo JU, Agarwal V, Guo H, Bartel DP. Expanded identification and characterization of mammalian circular RNAs. Genome Biol. 2014; 15: 409.

[10] Hansen TB, Jensen TI, Clausen BH, Bramsen JB, Finsen B, Damgaard CK, Kjems J. Natural RNA circles function as efficient microRNA sponges. Nature. 2013; 495: 384-8.

[11] Li Z, Huang C, Bao C, Chen L, Lin M, Wang X, Zhong G, Yu B, Hu W, Dai L, Zhu P, Chang Z, Wu Q, Zhao Y, Jia Y, Xu P, Liu H, Shan G. Exon-intron circular RNAs regulate transcription in the nucleus. Nat Struct Mol Biol. 2015; 22: 256-64.

[12] Ashwal-Fluss R, Meyer M, Pamudurti NR, Ivanov A, Bartok O, Hanan M, Evantal N, Memczak S, Rajewsky N, Kadener S. circRNA Biogenesis Competes with Pre-mRNA Splicing. Mol Cell. 2014; 56: 55-66.

[13] Bachmayr-Heyda A, Reiner AT, Auer K, Sukhbaatar N, Aust S, Bachleitner-Hofmann T, Mesteri I, Grunt TW, Zeillinger R, Pils D. Correlation of circular RNA abundance with proliferation - exemplified with colorectal and ovarian cancer, idiopathic lung fibrosis, and normal human tissues. Sci Rep. 2015; 5: 8057.

[14] Caiment F, Gaj S, Claessen S, Kleinjans J. High-throughput data integration of RNA-miRNA-circRNA reveals novel insights into mechanisms of benzo[a]pyrene-induced carcinogenicity. Nucleic Acids Res. 2015; 43: 2525-34.

[15] Westholm JO, Miura P, Olson S, Shenker S, Joseph B, Sanfilippo P, Celniker SE, Graveley BR, Lai EC. Genome-wide analysis of Drosophila circular RNAs reveals their structural and sequence properties and age-dependent neural accumulation. Cell Rep. 2014; 9: 1966-80.

[16] Bahn JH, Zhang Q, Li F, Chan TM, Lin X, Kim Y, Wong DT, Xiao X. The Landscape of microRNA, piwi-interacting RNA, and circular RNA in human saliva. Clin Chem. 2015; 61: 22130.

[17] Li P, Chen S, Chen H, Mo X, Li T, Shao Y, Xiao B, Guo J. Using circular RNA as a novel type of biomarker in the screening of gastric cancer. Clin Chim Acta. 2015; 444: 1326.

[18] Li Y, Zheng Q, Bao C, Li S, Guo W, Zhao J, Chen D, Gu J, He X, Huang S. Circular RNA is enriched and stable in exosomes: a promising biomarker for cancer diagnosis. Cell Res. 2015 Jul 3 [Epub ahead of print].

[19] Jemal A, Bray F, Center MM, Ferlay J, Ward E, Forman D. Global cancer statistics. CA Cancer J Clin. 2011; 61: 69-90.

[20] Sasaki Y, Yamada T, Tanaka H, Ohigashi H, Eguchi H, Yano $\mathrm{M}$, Ishikawa O, Imaoka S. Risk of recurrence in a long-term follow-up after surgery in 417 patients with hepatitis B- or hepatitis C-related hepatocellular carcinoma. Ann Surg. 2006; 244: 771-80.

[21] Bruix J, Gores GJ, Mazzaferro V. Hepatocellular carcinoma: clinical frontiers and perspectives. Gut. 2014 May; 63: 84455.

[22] Ghosal S, Das S, Sen R, Basak P, Chakrabarti J. Circ2Traits: 
A comprehensive database for circular RNA potentially associated with disease and traits. Front Genet. 2013; 4: 283.

[23] Ye QH, Qin LX, Forgues M, He P, Kim JW, Peng AC, Simon R, Li Y, Robles AI, Chen Y, Ma ZC, Wu ZQ, Ye SL, Liu YK, Tang ZY, Wang XW. Predicting hepatitis B viruspositive metastatic hepatocellular carcinomas using gene expression profiling and supervised machine learning. Nat Med. 2003; 9: 416-23.

[24] Li Y, Tang Y, Ye L, Liu B, Liu K, Chen J, Xue Q. Establishment of a hepatocellular carcinoma cell line with unique metastatic characteristics through in vivo selection and screening for metastasis-related genes through cDNA microarray. J Cancer Res Clin Oncol. 2003; 129: 43-51.

[25] Bi N, Cao J, Song Y, Shen J, Liu W, Fan J, He J, Shi Y, Zhang X, Lu N, Zhan Q, Wang L. A MicroRNA Signature Predicts Survival in Early Stage Small-Cell Lung Cancer Treated with Surgery and Adjuvant Chemotherapy. PLoS One. 2014; 9: e91388.

[26] Sze KM, Chu GK, Lee JM, Ng IO. C-terminal truncated hepatitis $\mathrm{B}$ virus $\mathrm{x}$ protein is associated with metastasis and enhances invasiveness by C-Jun/matrix metalloproteinase protein 10 activation in hepatocellular carcinoma. Hepatology. 2013; 57: 131-9.

[27] Chen X, Bo L, Zhao X, Chen Q. MicroRNA-133a inhibits cell proliferation, colony formation ability, migration and invasion by targeting matrix metallopeptidase 9 in hepatocellular carcinoma. Mol Med Rep. 2015; 11: 3900-7.

[28] McKenna GJ, Chen Y, Smith RM, Meneghetti A, Ong C, McMaster R, Scudamore CH, Chung SW. A role for matrix metalloproteinases and tumor host interaction in hepatocellular carcinomas. Am J Surg. 2002; 183: 588-94.

[29] Wang C, Jin G, Jin H, Wang N, Luo Q, Zhang Y, Gao D, Jiang K, Gu D, Shen Q, Huo X, Hu F, Ge T, Zhao F, Chu W, Shu H, Yao M, Cong W, Qin W. Clusterin facilitates metastasis by EIF3I/Akt/MMP13 signaling in hepatocellular carcinoma. Oncotarget. 2015; 6: 2903-16.

[30] Qu S, Yang X, Li X, Wang J, Gao Y, Shang R, Sun W, Dou K, Li H. Circular RNA: A new star of noncoding RNAs. Cancer Lett. 2015; 365: 141-8.

[31] Guo JU, Agarwal V, Guo H, Bartel DP. Expanded identification and characterization of mammalian circular RNAs. Genome Biol. 2014; 15: 409.

[32] Li JH, Liu S, Zhou H, Qu LH, Yang JH. starBase v2.0: decoding miRNA-ceRNA, miRNA-ncRNA and protein-RNA interaction networks from large-scale CLIP-Seq data. Nucleic Acids Res. 2014 Jan; 42(Database issue): D92-7.

[33] Thomas LF, Saetrom P. Circular RNAs are depleted of polymorphisms at microRNA binding sites. Bioinformatics. 2014; 30: $2243-6$.
[34] Weiß K, Antoniou A, Schratt G. Non-coding mechanisms of local mRNA translation in neuronal dendrites. Eur J Cell Biol. 2015 Jun 2 [Epub ahead of print].

\section{Supplementary material}

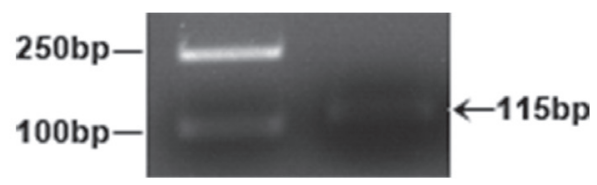

A

Melt Curve

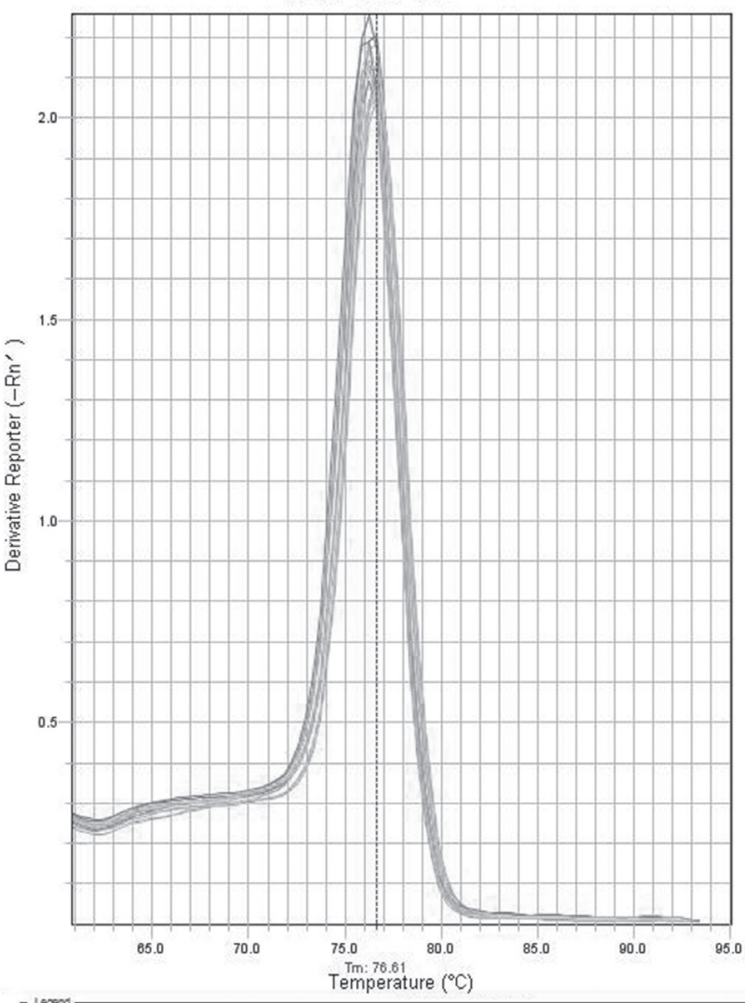

$\mathrm{A} \square \mathrm{B} \square \mathrm{C} \square \mathrm{D} \square \mathrm{E} \square \mathrm{F} \square \mathrm{G}$

B

Supplementary Fig. 1. The specificity of qRT-PCR product of hsa_circ_0001649 with divergent primers. (A) Electrophoresis of qRT-PCR product of hsa_circ_0001649. (B) Melting curve analysis of qRT-PCR product of hsa_circ_0001649. 